Dicle University Journal of Engineering (DUJE)

web: http://dergipark.gov.tr/dumf

Araştırma Makalesi / Research Article

\title{
Rogosinski Lemması ile ilgili Süren Nokta Empedans Fonksiyonları için Carathéodory Eşitsizliği
}

\section{Carathéodory's Inequality for Driving Point Impedance Functions Concerned with Rogosinski's lemma}

\section{Bülent Nafi Örnek ${ }^{1^{*}}$, Timur Düzenli}

${ }^{1}$ Amasya Üniversitesi, Bilgisayar Mühendisliği Bölümü, Amasya, nafiornek@gmail.com

${ }^{2}$ Amasya Üniversitesi, Elektrik Elektronik Mühendisliği Bölümü, Amasya, timur.duzenli@amasya.edu.tr

\begin{tabular}{l} 
MAKALE BİLGİLERİ \\
\hline Makale geçmişi: \\
Geliş: 1 Haziran 2020 \\
Düzeltme: 4 Ağustos 2020 \\
Kabul: 27 Ağustos 2020 \\
\hline Anahtar kelimeler: \\
Carathéodory eşitsizliği, Rogosinski \\
lemması, süren nokta empedans \\
fonksiyonu, pozitif reel fonksiyon
\end{tabular}

\begin{abstract}
ÖZET
Bu makalede, Carathéodory eşitsizliğinin bir sınır versiyonu, pozitif reel fonksiyonlar açısından incelenmiştir. Buna göre, $Z(s)$ süren nokta empedans fonksiyonu; s düzleminin sağ yarı düzleminde tanımlanmış, $Z(s)=\frac{A}{2}+$ $c_{1}(s-1)+c_{2}(s-1)^{2}+\cdots$ olarak verilen analitik bir fonksiyondur. $Z(s)$ fonksiyonunun sanal eksen üzerinde $\mathrm{s}$ $=0$ sınır noktasında da analitik olduğu varsayılarak, Rogosinski lemması yardımıyla, Z(s) 'nin türevinin modülü için yeni eșitsizlikler elde edilmiştir. Ayrıca, sunulan eşitsizliklerin kesinliği kanıtlanmıș ve elde edilen ekstremal fonksiyonların spektral özellikleri araştırılmıştır. Bu doğrultuda, çalışmada önerilen analizler kullanılarak çeşitli filtre yapılarının elde edilmesinin mümkün olduğu gözlenmiştir.
\end{abstract}

Doi: $10.24012 /$ dumf. 860229

\begin{tabular}{|c|c|}
\hline ARTICLE INFO & ABSTRACT \\
\hline Article history: & \multirow{5}{*}{$\begin{array}{l}\text { In this paper, a boundary version of the Carathéodory's inequality has been investigated for positive real functions. } \\
\text { Accordingly, the driving point impedance function } Z(s) \text { where } Z(s)=\frac{A}{2}+c_{1}(s-1)+c_{2}(s-1)^{2}+\ldots \text { is an } \\
\text { analytic function defined in the right half of the s-plane. With the help of Rogosinski' lemma, novel inequalities } \\
\text { have been derived for the modulus of derivative of } Z(s) \text { by assuming that the } Z(s) \text { function is also analytic at the } \\
\text { boundary point } s=0 \text { on the imaginary axis. In addition, the sharpness of presented inequalities has been proved } \\
\text { and the spectral characteristics of resulting extremal functions have been investigated. Accordingly, it has been } \\
\text { observed that it is possible to obtain various filter structures using proposed analysis in the study. }\end{array}$} \\
\hline Received: 1 June 2020 & \\
\hline Revised: 4 August 2020 & \\
\hline Accepted: 27 August 2020 & \\
\hline Keywords: & \\
\hline
\end{tabular}

Rogosinski's lemma, driving

point impedance function,

positive real function

* Sorumlu yazar / Correspondence author

Bülent Nafi ÖRNEK

$\triangle$ nafiornek@gmail.com 


\section{Giriș}

Pozitif reel fonksiyonlar (PRF), elektrik mühendisliğinde devre teorisinde [1], ăg sentezinde [2], kontrol sistemlerinde [3], sinyal işlemede [4] ve mikrodalga mühendisliğinde [5] sıklıkla kullanılmaktadır. Bir fonksiyonun pozitif reel olabilmesi için, aşağıdaki koşulları sağlamas1 gerekmektedir [6]:

$s$, karmaşık frekans değişkeni olmak üzere,

1-) $Z(s)$ analitik and $\mathfrak{R} s \geq 0$ 'da sanal eksen üzerindeki kutuplar hariç olmak üzere tek değerlidir,

2-) $Z(\bar{s})=\overline{Z(s)}$

3-) $\mathfrak{R} s \geq 0$ ' da $\mathfrak{R} Z(s) \geq 0$ olmalıdır.

$\mathrm{Bu}$ çalışmada, süren nokta empedans fonksiyonlarının (SNEF) türevi için bir sınır analizi yapılması amaçlanmıştır. SNEF'lerin türevi jiroskop tasarımı [7], doğrusal karşılıklı sistemlerin yorumlanması [8] gibi çeşitli amaçlar için kullanılabilmektedir.

Burada, $Z(s)$ süren nokta empedans fonksiyonunun türevinin modülünün sıfır noktasındaki değeri, yani $\left|Z^{\prime}(0)\right|$ göz önüne alınarak üç teorem içerisinde $Z^{\prime}(s)$ için üç alt sinır sunulmaktadır. [9] ve [10] ile benzer şekilde, bu çalışmanın amacı $Z(s)$ fonksiyonunun türevinin sınırdaki davranışını anlamaktır. Burada sunulan çalışmanın öncekilerden temel farkı, yeni sınırları elde etmek için Rogosinski Lemması ile beraber Carathéodory Eşitsizliği kullanılmasıdır. Ayrıca, $Z$ (s) fonksiyonunun türevinin analizi, çalışmada ele alınan problemin doğal bir sonucudur. Sunulan analizlerle, Carathéodory Eşitsizliği ile SNEF'lerin arasındaki ilişkinin ortaya konulması hedeflenmiştir. Bu ilişkiyi somutlaştırmak adına, devre teorisinde sıkça kullanılan ekstremal fonksiyonlar olan SNEF'lerden faydalanılmıştır.

Elde edilen sonuçlara göre, Rogosinski Lemması ile beraber Carathéodory Eşitsizliği'nin SNEF'lere uygulanmasıyla çok çentikli süzgeçler de dahil olmak üzere çeşitli süzgeç yapıları oluştuğunu söylemek mümkündür.

\section{Ön Değerlendirmeler}

$f(z)$, birim disk içerisinde $(U=\{z:|z|<1\})$ analitik bir fonksiyon olsun. Maksimum Prensibi'nin bir sonucu olan Schwarz Önermesi'ne göre, $f: U \rightarrow U$ fonksiyonu $f(0)=$ 0 ile analitik olduğu durumda, her $z \in U$ için $|f(z)| \leq|z|$ ve $\left|f^{\prime}(0)\right| \leq 1^{\prime}$ dir. Ayrıca, $z \neq 0$ için $|f(z)|=|z|$ geçerli olduğu takdirde veya $\left|f^{\prime}(0)\right|=1$ ise $f$ bir rotasyondur. Başka bir ifadeyle, $\theta$ reel olmak üzere $f(z)=z e^{i \theta}$, dir [11].

Bunun kesin bir versiyonu Rogosinski Lemması'dır [12,13]. Rogosinski Lemması'na göre, bütün $z \in U$ değerleri için $a_{1}=$ $\frac{z f^{\prime}(0)\left(1-|z|^{2}\right)}{1-|z|^{2}\left|f^{\prime}(0)\right|^{2}}$ ve $r_{1}=\frac{|z|^{2}\left(1-\left|f^{\prime}(0)\right|^{2}\right)}{1-|z|^{2}\left|f^{\prime}(0)\right|^{2}}$ olmak üzere $\left|f(z)-a_{1}\right| \leq r_{1}$ 'dir.

Carathéodory'nin Eșitsizliği, literatürdeki çeșitli çalışmalarda ele alınmıștır. Carathéodory Eşitsizliği'nin tam fonksiyonlar ve analitik fonksiyonlar teorisindeki rolü kapsamlı bir şekilde tartışılmıştır [14]. Ayrıca, Carathéodory Eşitsizliği'nin birim diskteki eșitsizliğinin bir sınır versiyonu da [15] ve [16] çalışmalarında dikkate alınmakta ve yeni sonuçlar elde edilmektedir. Mercer [12], Schwarz Lemması'nın, iki noktanın görüntülerinin bilindiği bir versiyonunu kanıtlamıştır. Ayrıca; Mercer, sınırdaki bazı Schwarz ve Carathéodory eşitsizliklerini, Rogosinski'ye bağlı bir lemmanın sonucu olarak görmektedir [13]. Buna ek olarak, birim diski kendisine eşleyen analitik fonksiyonlar için yeni bir sınır Schwarz Lemması elde etmiştir [17].

İlk olarak, Carathéodory'nin pozitif reel fonksiyonlar için sağ yarım düzlemdeki eşitsizliği sunulacaktır.

$Z(s)=\frac{A}{2}+c_{1}(s-1)+c_{2}(s-1)^{2}+\cdots$ pozitif reel bir fonksiyon ve $\mathfrak{R} s \geq 0$ 'da $0<\Re Z(s) \leq A$ olsun. $f(z), U$ içerisinde analitik bir fonksiyon, $f(0)=0$ ve $|z|<1$ için $|f(z)|<1$ olmak üzere

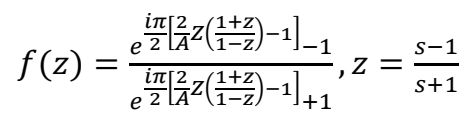

fonksiyonunu göz önüne alalım.

(1) denkleminden 


$$
f^{\prime}(z)=\frac{\frac{4 i \pi}{A(1-z)^{2}} Z^{\prime}\left(\frac{1+z}{1-z}\right) e^{\frac{i \pi}{2}\left[\frac{2}{A} Z\left(\frac{1+z}{1-z}\right)-1\right]}}{\left(e^{\frac{i \pi}{2}\left[\frac{2}{A} Z\left(\frac{1+z}{1-z}\right)-1\right]}+1\right)^{2}}
$$

sonucu bulunur. Dolayısıyla, Schwarz Lemması'nı $f^{\prime}(z)$ için uygulayarak

$$
\left|f^{\prime}(0)\right|=\left|\frac{\frac{4 i \pi}{A} Z^{\prime}(1) e^{\frac{i \pi}{2}\left[\frac{2}{A} Z(1)-1\right]}}{\left(e^{\frac{i \pi}{2}\left[\frac{2}{A} Z(1)-1\right]}+1\right)^{2}}\right| \leq 1
$$

ve

$$
\left|Z^{\prime}(1)\right| \leq \frac{A}{\pi}
$$

eşitsizliği elde edilir.

Şimdi bu eşitsizliğin eşitlik halini gösterelim.

$$
Z(s)=\frac{A}{2}\left(1+\frac{2}{i \pi} \ln s\right)
$$

olsun. Bu durumda,

$$
\begin{aligned}
\frac{A}{2}+c_{1}(s-1)+ & c_{2}(s-1)^{2}+\cdots \\
& =\frac{A}{2}\left(1+\frac{2}{i \pi} \ln s\right), \\
c_{1}(s-1) & +c_{2}(s-1)^{2}+\cdots=\frac{A}{i \pi} \ln s
\end{aligned}
$$

ve

$$
c_{1}+c_{2}(s-1)+\cdots=\frac{A}{i \pi} \frac{\ln s}{s-1} .
$$

Son ifadede $s \rightarrow 1$ için limite geçersek

$$
\left|c_{1}\right|=\frac{A}{\pi}
$$

elde ederiz. Bu da

$$
\left|Z^{\prime}(1)\right|=\frac{A}{\pi}
$$

olduğunu gösterir.

Dolayısıyla, aşağıdaki lemma elde edilir:

Lemma 1. $Z(s)=\frac{A}{2}+c_{1}(s-1)+c_{2}(s-$ $1)^{2}+\cdots$ fonksiyonu $\mathfrak{R} s \geq 0$ 'da $0<\Re Z(s) \leq$ $A$ için pozitif reel bir fonksiyon olsun. $\mathrm{Bu}$ durumda aşağıdaki eşitsizlik elde edilir:

$$
\left|Z^{\prime}(1)\right| \leq \frac{A}{\pi}
$$

Eşitsizlik (2) kesindir ve ekstremal fonksiyon aşağıdaki gibi verilir:

$$
Z(s)=\frac{A}{2}\left(1+\frac{2}{i \pi} \ln s\right) .
$$

$\mathrm{Bu}$ sonuç, sağ yarı düzlemdeki pozitif reel fonksiyon için Carathéodory Eşitsizliği'ni doğurmaktadır.

Şekil l'de, Lemma 1'de elde edilen SNEF'nin büyüklüğü frekansa bağlı olarak ve $A=1$ olduğu varsayılarak gösterilmiştir. Şekilde görülebileceği gibi $A$ parametresi, SNEF'nin alabileceği en düşük empedans değerini belirleyen parametredir. Şekil 1'dekine benzer bir frekans karakteristiğini seri olarak bağlanmış bir LC devre yapısı elde etmek mümkündür.

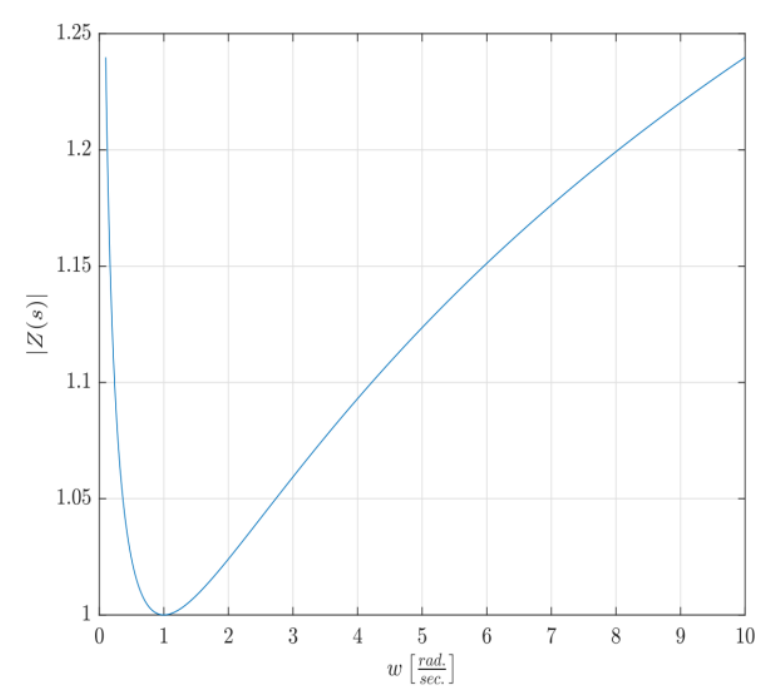

Şekil 1. Lemma l'de elde edilen $Z(s)=$ $\frac{A}{2}\left(1+\frac{2}{i \pi} \operatorname{lns}\right)$ fonksiyonu için frekans yanitı.

Schwarz Lemması'nın önemli bir sonucu, Osserman tarafindan aşağıdaki şekilde ifade edilmiştir [18]:

$f, U$ dairesinde analitik, $f(0)=0$ ve $|z|<1$ için $\mid f(z)) \mid<1$ olsun. Ayrica varsayalım ki, $f$ fonksiyonu bir $z_{0} \in \partial U=\{z:|z|=1\}$ noktasina sürekli devam ediliyor, $\left|f\left(z_{0}\right)\right|=1$, ve $f^{\prime}\left(z_{0}\right)$ mevcuttur. $\mathrm{O}$ zaman

$$
\left|f^{\prime}\left(z_{0}\right)\right| \geq \frac{2}{1+\left|f^{\prime}(0)\right|} \geq 1
$$

eşitsizliği sağlanır.

(3) eşitsizliğinde, eşitlik hali $\left(z_{0}=1\right.$ olduğunda) $f(z)=z \frac{z+m}{1+m z}, 0 \leq m \leq 1$ fonksiyonu için gerçeklenir. (1.3) eşitsizliğinin ikinci kısmının eşitlik hali $f(z)=z e^{i \alpha}, \alpha$ reel olduğunda 
mümkündür. (3) eşitsizliği ve genellemeleri geometrik fonksiyonlar teorisinde önemli uygulamalara sahiptir. [18, 19, 20, 21, 22].

\section{Ana Sonuçlar}

$\mathrm{Bu}$ kısımda, pozitif reel fonksiyonların sinır analizi sonuçları sunulmaktadır. Pozitif reel fonksiyonların tanımından, $Z(s)$ fonksiyonu analitik ve s-düzleminin sağ yarısında tekil değerli olarak ifade edilebilir. Așağıdaki teoremde, $Z(0)=A$ olmakla birlikte, pozitif reel fonksiyonlar için $Z(0)$ 'in türevinin üzerinde aşağı sınırlar oluşturulmaktadır.

Teorem 1. $Z(s)=\frac{A}{2}+c_{1}(s-1)+c_{2}(s-$ $1)^{2}+\ldots$ fonksiyonu, $\Re s^{2} \geq 0$ için $0<\Re Z(s) \leq$ $A$ olan ve $Z(0)=A$ ile sanal eksenin $s=0$ noktasında da analitik olan pozitif reel bir fonksiyon olsun. Bu durumda,

$$
\left|Z^{\prime}(0)\right| \geq \frac{2 A}{\pi}
$$

(4) sonucu aşağıdaki fonksiyon için kesindir.

$$
Z(s)=\frac{A}{2}\left(1+\frac{2}{i \pi} \ln \left(\frac{(1-i) s+1+i}{(1+i) s+1-i}\right)\right) .
$$

İspat. Aşağıdaki $f(z)$ fonksiyonunu göz önüne alalım.

$$
f(z)=\frac{e^{\frac{i \pi}{2}\left[\frac{2}{A} z\left(\frac{1+z}{1-z}\right)-1\right]}-1}{e^{\frac{i \pi}{2}\left[\frac{2}{A} z\left(\frac{1+z}{1-z}\right)-1\right]}+1} .
$$

$f(z)$ fonksiyonu $U$ içerisinde analitik bir fonksiyondur, $f(0)=0$ 'dır ve $|z|<1$ için $|f(z)|<1$ 'dir. Ayrıca, $s=0$ için (sanal eksenin $s=0$ noktasi)

$$
f(-1)=\frac{e^{\frac{i \pi}{2}\left[\frac{2}{A} Z(0)-1\right]}-1}{e^{\frac{i \pi}{2}\left[\frac{2}{A} Z(0)-1\right]}+1}=\frac{e^{\frac{i \pi}{2}}-1}{e^{\frac{i \pi}{2}}+1}=\frac{i-1}{i+1}, Z(0)=A
$$

ve

$$
|f(-1)|=1 .
$$

sonuçlarına ulaşılır.

$f(z)$ 'in tanımından, basit hesaplamalarla

$$
f^{\prime}(z)=\frac{\frac{4 i \pi}{A(1-z)^{2}} Z^{\prime}\left(\frac{1+z}{1-z}\right) e^{\frac{i \pi}{2}\left[\frac{2}{A} Z\left(\frac{1+z}{1-z}\right)-1\right]}}{\left(e^{\frac{i \pi}{2}\left[\frac{2}{A} Z\left(\frac{1+z}{1-z}\right)-1\right]}+1\right)^{2}}
$$

sonucunu elde ederiz. Dolayısıyla, $z_{0}=-1 \in$ $\partial U$ için $\left|f^{\prime}\left(z_{0}\right)\right| \geq 1$ olduğundan

$$
\begin{aligned}
1 & \leq\left|f^{\prime}(-1)\right|=\left|\frac{\frac{4 i \pi}{A 4} Z^{\prime}(0) e^{\frac{i \pi}{2}\left[\frac{2}{A} Z(0)-1\right]}}{\left(e^{\frac{i \pi}{2}}\left[\frac{2}{A} Z(0)-1\right]+1\right)^{2}}\right| \\
& =\left|\frac{\frac{i \pi}{A} Z^{\prime}(0) e^{\frac{i \pi}{2}}}{\left(e^{\frac{i \pi}{2}}+1\right)^{2}}\right|=\frac{\pi}{A} \frac{\left|Z^{\prime}(0)\right|}{\left|e^{\frac{i \pi}{2}}+1\right|^{2}}
\end{aligned}
$$

$\left|e^{\frac{i \pi}{2}}+1\right|^{2}=|i+1|^{2}=2$ olduğundan

$$
\left|Z^{\prime}(0)\right| \geq \frac{2 A}{\pi}
$$

elde edilir.

Şimdi, (4) eşitsizliğinin kesin olduğu gösterilecektir.

$Z(s)=\frac{A}{2}\left(1+\frac{2}{i \pi} \ln \left(\frac{(1-i) s+1+i}{(1+i) s+1-i}\right)\right) \quad$ olsun. $\mathrm{Bu}$ durumda,

$$
\begin{aligned}
& Z^{\prime}(s) \\
& =\frac{A}{i \pi} \frac{(1-i)((1+i) s+1-i)}{((1+i) s+1-i)(1-i) s+1+i} \\
& -\frac{A}{i \pi} \frac{(1+i)((1-i) s+1+i)}{((1+i) s+1-i)(1-i) s+1+i}
\end{aligned}
$$

ve

$$
\left|Z^{\prime}(0)\right|=\frac{2 A}{\pi}
$$

olur.

Teorem 1'de elde edilen SNEF'in büyüklüğünün frekansa göre çizimi $A=1$ varsayımı yapılarak Şekil 2'de gösterilmektedir. Şekil 1'den farklı olarak, Şekil 2'de en yüksek empedans değerini belirleyen parametre $A$ parametresidir. Şekil 2 'deki grafiği, paralel bağlanmış bir LC devresi ile yaklaşık olarak elde etmek mümkündür. 


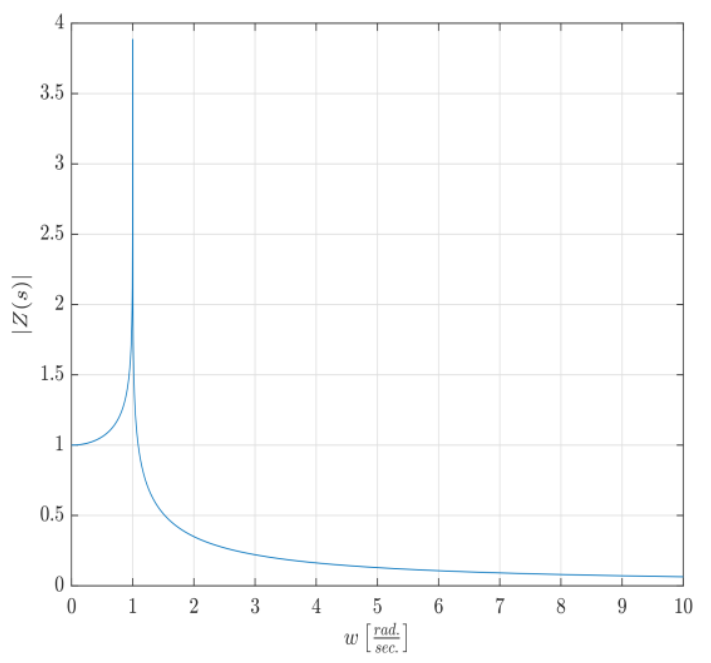

Şekil 2. Teorem l'de elde edilen $Z(s)=\frac{A}{2}(1+\quad \mathrm{ve}$

$\left.\frac{2}{i \pi} \ln \left(\frac{(1-i) s+1+i}{(1+i) s+1-i}\right)\right)$ fonksiyonu için frekans yanitı.

(4) eşitsizliği, $Z(s)$ fonksiyonunun Taylor açılımındaki ilk katsayı olan $\left|Z^{\prime}(1)\right|$ 'i dikkate alarak aşağıdan kuvvetlendirilebilir.

Teorem 2. Teorem 1 ile aynı varsayımlar altında

$$
\left|Z^{\prime}(0)\right| \geq \frac{4 A^{2}}{\pi\left(A+\pi\left|Z^{\prime}(1)\right|\right)}
$$

olur. Buna ek olarak, (2.2) eşitsizliği aşağıda verilen fonksiyon için kesindir:

$Z(s)$

$$
=\frac{A}{2}\left(1 \frac{2}{i \pi} \ln \left(\frac{1-a \frac{s-1}{s+1}+i\left(\frac{s-1}{s+1}\right)^{2}-i a \frac{s-1}{s+1}}{1-a \frac{s-1}{s+1}-i\left(\frac{s-1}{s+1}\right)^{2}+i a \frac{s-1}{s+1}}\right)\right) .
$$

Burada, $a$ katsayısı $a=\frac{\pi}{A}\left|Z^{\prime}(1)\right|$ olarak verilen $(0,1]$ aralığında değer alan keyfi bir sayıdır $((2)$ eşitsizliğine bakınız).

İspat. $f(z)$ fonksiyonu Teorem 1 'in ispatındakiyle aynı fonksiyon olsun. Rogosinski Lemması'ndan $a_{1}=\frac{z f^{\prime}(0)\left(1-|z|^{2}\right)}{1-|z|^{2}\left|f^{\prime}(0)\right|^{2}} \quad$ ve $\quad r_{1}=$ $\frac{|z|^{2}\left(1-\left|f^{\prime}(0)\right|^{2}\right)}{1-|z|^{2}\left|f^{\prime}(0)\right|^{2}}$ olmak üzere $\left|f(z)-a_{1}\right| \leq r_{1}$ 'dir.

Dolayısıyla,

$$
\begin{aligned}
& \left|\frac{f(z)-1}{z-1}\right| \geq \frac{1-\left|a_{1}\right|-r_{1}}{1-|z|} \\
& =\frac{1-\frac{|z|\left|f^{\prime}(0)\right|\left(1-|z|^{2}\right)}{1-|z|^{2}\left|f^{\prime}(0)\right|^{2}}-\frac{|z|^{2}\left(1-\left|f^{\prime}(0)\right|^{2}\right)}{1-|z|^{2}\left|f^{\prime}(0)\right|^{2}}}{1-|z|}
\end{aligned}
$$

$$
\begin{gathered}
=\frac{1-|z|^{2}\left|f^{\prime}(0)\right|^{2}-|z|\left|f^{\prime}(0)\right|\left(1-|z|^{2}\right)-|z|^{2}\left(1-\left|f^{\prime}(0)\right|^{2}\right)}{(1-|z|)\left(1-|z|^{2}\left|f^{\prime}(0)\right|^{2}\right)} \\
=\frac{1+|z|}{1+|z|\left|f^{\prime}(0)\right|}
\end{gathered}
$$

olur.

Son eşitsizlikte limite geçersek

$$
\left|f^{\prime}(-1)\right| \geq \frac{2}{1+\left|f^{\prime}(0)\right|}
$$

sonucunu elde ederiz. $\left|f^{\prime}(-1)\right|=\frac{\pi}{A} \frac{\left|z^{\prime}(0)\right|}{2}$ ve $\left|f^{\prime}(0)\right|=\frac{\pi\left|Z^{\prime}(1)\right|}{A}$ olduğundan

$$
\frac{\pi}{A} \frac{\left|Z^{\prime}(0)\right|}{2} \geq \frac{2}{1+\frac{\pi\left|Z^{\prime}(1)\right|}{A}}
$$

\section{e}

$$
\left|Z^{\prime}(0)\right| \geq \frac{4 A^{2}}{\pi\left(A+\pi\left|Z^{\prime}(1)\right|\right)}
$$

eşitsizliklerine ulaşılır.

Şimdi, (5) eşitsizliğinin kesin olduğu gösterilecektir.

$Z\left(\frac{1+z}{1-z}\right)=\frac{A}{2}\left(1+\frac{2}{i \pi} \ln \left(\frac{1-a z+i z^{2}-i a z}{1-a z-i z^{2}+i a z}\right)\right) \quad$ olsun. $\mathrm{Bu}$ denklemden, $\left|Z^{\prime}(0)\right|=\frac{4 A}{\pi(1+a)}$ olduğu görülür. $a=$ $\frac{\pi}{A}\left|Z^{\prime}(1)\right|$ olduğundan, (5) eşitsizliği eşitlikle sağlanır.

Teorem 2'de elde edilen SNEF'in frekans karakteristiği Şekil 3'de gösterilmektedir. Şekil 3'e göre, SNEF $0 \leq w \leq 8 \mathrm{rad} / \mathrm{sn}$. aralığında iki kutup ve bir sifira sahiptir. Diğer yanda; SNEF, $w>8 \mathrm{rad} / \mathrm{sn}$ için, $A$ değerinde bir direnç gibi davranmaktadır (şekilde $A=1$ olduğu varsayılmıştır). 


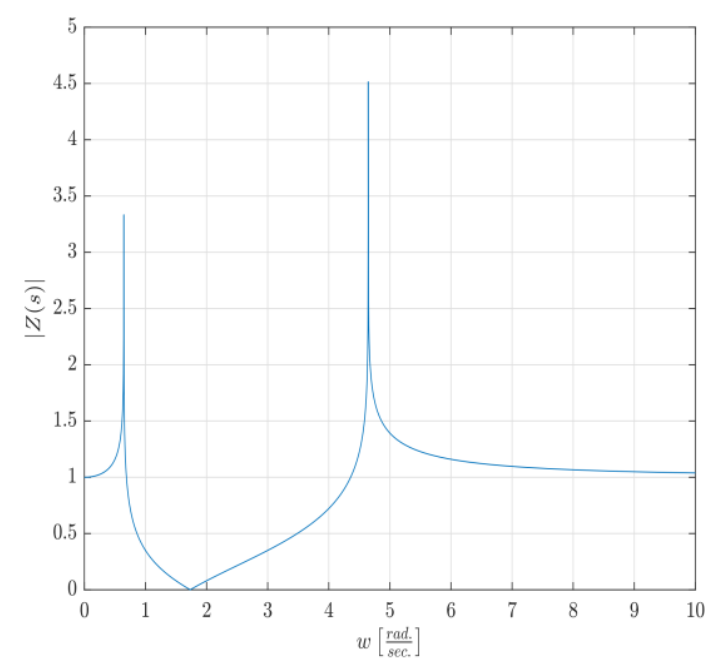

Şekil 3. Teorem 2'de elde edilen $Z(s)=\frac{A}{2}(1+$ $\left.\frac{2}{i \pi} \ln \left(\frac{(1-i) s+1+i}{(1+i) s+1-i}\right)\right)$ fonksiyonu için frekans yanitt.

Aşağıdaki teoremde, $Z(s)$ fonksiyonunun $c_{1}$ ve $c_{2}$ ardışık katsayıları eklenerek (5) eşitsizliği kuvvetlendirilmiştir.

Teorem 3.3. $Z(s)=\frac{A}{2}+c_{1}(s-1)+c_{2}(s-1)^{2}+\ldots$ fonksiyonu, $\Re s \geq 0$ için $0<\Re Z(s) \leq A$ olan ve $Z(0)=A$ ile sanal eksenin $s=0$ noktasinda da analitik olan pozitif reel bir fonksiyon olsun. $\mathrm{Bu}$ durumda,

$$
\left|Z^{\prime}(0)\right| \geq \frac{2 A}{\pi}\left(1+\frac{2\left(A-\pi\left|c_{1}\right|\right)^{2}}{A^{2}-\pi^{2}\left|c_{1}\right|^{2}+A \pi\left|c_{1}+2 c_{2}\right|}\right)
$$

olur.

(6) eşitsizliği aşağıda verilen ekstremal fonksiyon için kesindir:

$$
Z(s)=\frac{A}{2}\left(1+\frac{2}{i \pi} \ln \left(\frac{(s+1)^{2}+i(s-1)^{2}}{(s+1)^{2}-i(s-1)^{2}}\right)\right)
$$

İspat. $f(z)$ Teorem 1 'deki ile aynı fonksiyon olduğunu varsayalım. $\quad r(z)=\frac{f(z)}{z} \quad$ ve $\quad v(z)=$ $\frac{r(z)-r(0)}{1-\overline{r(0) r(z)}}$ fonksiyonlarını göz önüne alalım. Burada; $v(z), U$ içerisinde analitik, $v(0)=0$ ve $|v(z)|<1$ for $z \in U$ olmak üzere,

$$
v^{\prime}(0)=\frac{r^{\prime}(0)}{1-|r(0)|^{2}}=\frac{f^{\prime \prime}(0)}{2\left(1-\left|f^{\prime}(0)\right|^{2}\right)} \text {. }
$$

Rogosinski Lemması ve [12], [13]'den

$$
\left|f(z)-a_{2}\right| \leq r_{2}
$$

sonucuna ulaşılır. Burada, $a_{2}=\frac{z\left|f^{\prime}(0)\right|\left(1-\kappa^{2}\right)}{1-\kappa^{2}\left|f^{\prime}(0)\right|^{2}}, r_{2}=$ $\frac{\kappa|z|\left(1-\left|f^{\prime}(0)\right|^{2}\right)}{1-\kappa^{2}\left|f^{\prime}(0)\right|^{2}} \quad$ ve $\quad \kappa=|z| \frac{|z|+\left|v^{\prime}(0)\right|}{1+|z|\left|v^{\prime}(0)\right|} \quad$ olarak verilmektedir. Dolayısıyla,

$$
\begin{aligned}
& \left|\frac{f(z)-1}{z-1}\right| \geq \frac{1-\left|a_{2}\right|-r_{2}}{1-|z|} \\
& =\frac{1-\frac{|z|\left|f^{\prime}(0)\right|\left(1-\kappa^{2}\right)}{1-\kappa^{2}\left|f^{\prime}(0)\right|^{2}}-\frac{\kappa|z|\left(1-\left|f^{\prime}(0)\right|^{2}\right)}{1-\kappa^{2}\left|f^{\prime}(0)\right|^{2}}}{1-|z|} \\
& =\frac{1-\kappa^{2}\left|f^{\prime}(0)\right|^{2}-|z|\left|f^{\prime}(0)\right|\left(1-\kappa^{2}\right)-\kappa|z|\left(1-\left|f^{\prime}(0)\right|^{2}\right)}{(1-|z|)\left(1-\kappa^{2}\left|f^{\prime}(0)\right|^{2}\right)} \\
& =\frac{1+\kappa\left|f^{\prime}(0)\right|-|z|\left|f^{\prime}(0)\right|-\kappa|z|}{(1-|z|)\left(1+\kappa\left|f^{\prime}(0)\right|\right)}
\end{aligned}
$$

sonucu elde edilir.

$\kappa=|z| \frac{|z|+\left|v^{\prime}(0)\right|}{1+|z|\left|v^{\prime}(0)\right|}$ olduğundan,

$$
\begin{aligned}
& \left|\frac{f(z)-1}{z-1}\right| \\
& \geq \frac{1+|z| \frac{|z|+\left|v^{\prime}(0)\right|}{1+|z|\left|v^{\prime}(0)\right|}\left|f^{\prime}(0)\right|-|z|\left|f^{\prime}(0)\right|-|z| \frac{|z|+\left|v^{\prime}(0)\right|}{1+|z|\left|v^{\prime}(0)\right|}|z|}{(1-|z|)\left(1+|z| \frac{|z|+\left|v^{\prime}(0)\right|}{1+|z|\left|v^{\prime}(0)\right|}\left|f^{\prime}(0)\right|\right)} \\
& =\frac{1+|z|+|z|^{2}+|z|\left|v^{\prime}(0)\right|(1-|z|)}{1+|z|\left|v^{\prime}(0)\right|+|z|^{2}\left|f^{\prime}(0)\right|+|z|\left|f^{\prime}(0)\right|\left|v^{\prime}(0)\right|} \\
& -\frac{|z|\left|f^{\prime}(0)\right|(1-|z|)+|z|\left|f^{\prime}(0)\right|\left|v^{\prime}(0)\right|(1-|z|)}{1+|z|\left|v^{\prime}(0)\right|+|z|^{2}\left|f^{\prime}(0)\right|+|z|\left|f^{\prime}(0)\right|\left|v^{\prime}(0)\right|} .
\end{aligned}
$$

sonucuna ulaşırız. Son eşitsizlikte limite geçilirse

$$
\begin{aligned}
& \left|f^{\prime}(-1)\right| \geq \frac{3+\left|v^{\prime}(0)\right|-\left|f^{\prime}(0)\right|+\left|f^{\prime}(0)\right|\left|v^{\prime}(0)\right|}{1+\left|v^{\prime}(0)\right|+\left|f^{\prime}(0)\right|+\left|f^{\prime}(0)\right|\left|v^{\prime}(0)\right|} \\
& =\frac{3+\left|v^{\prime}(0)\right|-\left|f^{\prime}(0)\right|+\left|f^{\prime}(0)\right|\left|v^{\prime}(0)\right|}{\left(1+\left|v^{\prime}(0)\right|\right)\left(1+\left|f^{\prime}(0)\right|\right)}
\end{aligned}
$$

olur. Bazı küçük değişiklikler yapılarak

$$
\begin{aligned}
\left|f^{\prime}(-1)\right| \geq 1+ & \frac{2\left(1-\left|f^{\prime}(0)\right|\right)^{2}}{\left(1+\left|v^{\prime}(0)\right|\right)\left(1-\left|f^{\prime}(0)\right|^{2}\right)} \\
& =1+\frac{4\left(1-\left|f^{\prime}(0)\right|\right)^{2}}{2\left(1-\left|f^{\prime}(0)\right|^{2}\right)+\left|f^{\prime \prime}(0)\right|}
\end{aligned}
$$

elde edilir. $\left|f^{\prime}(-1)\right|=\frac{\pi}{A} \frac{\left|Z^{\prime}(0)\right|}{2},\left|f^{\prime}(0)\right|=\frac{\pi\left|c_{1}\right|}{A} \quad$ ve $\left|f^{\prime \prime}(0)\right|=\frac{2 \pi}{A}\left|c_{1}+2 c_{2}\right|$ olduğundan

$$
\frac{\pi}{A} \frac{\left|Z^{\prime}(0)\right|}{2} \geq 1+\frac{4\left(1-\frac{\pi\left|c_{1}\right|}{A}\right)^{2}}{2\left(1-\left(\frac{\pi\left|c_{1}\right|}{A}\right)^{2}\right)+\frac{2 \pi}{A}\left|c_{1}+2 c_{2}\right|}
$$

ve

$$
\left|Z^{\prime}(0)\right| \geq \frac{2 A}{\pi}\left(1+\frac{2\left(A-\pi\left|c_{1}\right|\right)^{2}}{A^{2}-\pi^{2}\left|c_{1}\right|^{2}+A \pi\left|c_{1}+2 c_{2}\right|}\right)
$$


olur. Şimdi, (6) eşitsizliğinin kesin olduğu gösterilecektir. Aşağıdaki fonksiyona bakalım:

$\mathrm{Z}\left(\frac{1+z}{1-z}\right)=\frac{A}{2}\left(1+\frac{2}{i \pi} \ln \left(\frac{1+\mathrm{i} z^{2}}{1-\mathrm{i} z^{2}}\right)\right)$.

Dolayısıyla, $\left|Z^{\prime}(0)\right|=\frac{4 A}{\pi}$, dir.

Diğer yandan,

$$
\begin{aligned}
& \frac{A}{2}+c_{1} \frac{2 z}{1-z}+ c_{2}\left(\frac{2 z}{1-z}\right)^{2}+\ldots \\
&=\frac{A}{2}\left(1+\frac{2}{i \pi} \ln \left(\frac{1+i z^{2}}{1-i z^{2}}\right)\right), \\
& c_{1} \frac{2 z}{1-z}+c_{2}\left(\frac{2 z}{1-z}\right)^{2}+\cdots=\frac{A}{i \pi} \ln \left(\frac{1+i z^{2}}{1-i z^{2}}\right)
\end{aligned}
$$

sonuçlarına ulaşı1ır. Limit durumuna geçildiğinde son denklem, $c_{1}=0$ olduğunu ifade etmektedir. Benzer şekilde, basit hesaplamalarla $c_{2}=\frac{A}{2 \pi}$ olduğu görülebilir. Bu yüzden,

$$
\begin{array}{r}
\frac{2 A}{\pi}\left(1+\frac{2\left(A-\pi\left|c_{1}\right|\right)^{2}}{A^{2}-\pi^{2}\left|c_{1}\right|^{2}+A \pi\left|c_{1}+2 c_{2}\right|}\right) \\
=\frac{2 A}{\pi}\left(1+\frac{2 A^{2}}{A^{2}+A^{2}}\right)=\frac{4 A}{\pi}
\end{array}
$$

sonucuna ulaşılır.

Şekil 3'tekine benzer bir frekans yanıtı burada da elde edilmektedir. $\mathrm{Bu}$ yanıt, Şekil 4'te gösterilmektedir. Teorem 2'deki gibi, burada da frekans yanıtı $5 \mathrm{rad} / \mathrm{sn}$ 'den büyük frekans değerleri için dirençsel (rezistif) bir yap1 göstermektedir. Daha önce sunulan teoremlerde olduğu gibi, direncin değeri $A$ parametresine bağlıdır.

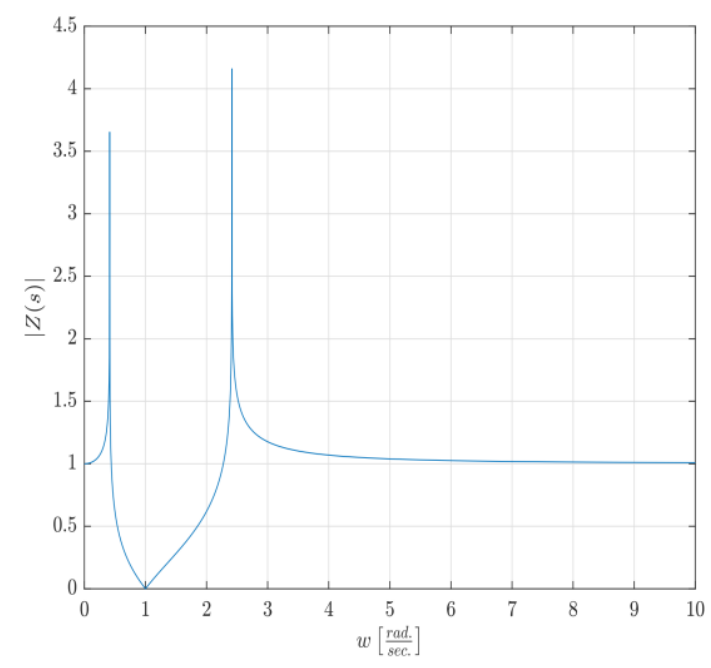

Şekil 4. Teorem 3'te elde edilen $Z(s)=\frac{A}{2}(1+$ $\left.\frac{2}{i \pi} \ln \left(\frac{(s+1)^{2}+i(s-1)^{2}}{(s+1)^{2}-i(s-1)^{2}}\right)\right)$ fonksiyonu için frekans yanıtt.

\section{Tartışma}

$\mathrm{Bu}$ çalışmada, süren nokta empedans fonksiyonlarının sınır analizi yapılmıştır. Buna göre, Rogosinski Lemması ile beraber Carathéodory Eşitsizliği'ni kullanarak $|Z(s)|$ için alt sınırlar belirleyen üç teorem sunulmuştur. Teorem 1'de verilen eşitsizlik, $\left|Z^{\prime}(1)\right|$ teriminin eklenmesiyle Teorem 2'de güçlendirilmiştir. Benzer şekilde, $\operatorname{ard}$ şık $c_{1}$ ve $c_{2}$ katsayılarını hesaba katarak Teorem 2 kuvvetlendirilmiş ve Teorem 3 'te ortaya yeni bir sınır konmuştur. Elde edilen bütün eşitsizlikler, kesinlik analizine tabi tutulmuş ve bu sayede her teorem için ekstremal fonksiyonlar elde edilmiştir. Elde edilen bu ekstremal fonksiyonların, frekans karakteristiği grafiklerine göre, bu çalışmada önerilen teoremlerle farklı özellikte süzgeç (filtre) yapıları tasarlamak mümkündür.

\section{Kaynaklar}

[1] Örnek, B. N., Düzenli, T., (2019). Pozitif Reel Fonksiyonlar için Devre Uygulamaları. DÜMF Mühendislik Dergisi, 10, 2, 457-465.

[2] Sharma, A., Soni, T., (2017). A review on passive network synthesis using Cauer form. World Journal of Wireless Devices and Engineering, 1, 1, 39-46.

[3] Ishida, M., Fukui, Y., \& Ebisutani, K., (1984). Novel active-R synthesis of a driving-point impedance. International Journal of Electronics, 56, 1, 151-158.

[4] Ochoa, A., (2016). Driving point impedance and signal flow graph basics: a systematic approach to circuit analysis. In Feedback in analog circuits (pp. 13-34). Springer, Cham.

[5] Wunsch, A. D., Hu, S. P., (1996). A closed-form expression for the driving-point impedance of the small inverted L antenna. IEEE Transactions on Antennas and Propagation, 44, 2, 236-242.

[6] Richards, P. I., (1947). A special class of functions with positive real part in a half-plane. Duke Mathematical Journal, 14, 3, 777-786.

[7] Hazony, D., (1963). Elements of network synthesis. Reinhold, NY, USA.

[8] Reza, F. M., (1961). Schwarz's lemma and linear passive systems. Proc. IRE, 49, 2, 17-23.

[9] Örnek, B. N., Düzenli, T., (2019). On boundary 
analysis for derivative of driving point impedance functions and its circuit applications. IET Circuits, Devices \& Systems, 13, 2, 145-152.

[10] Örnek, B. N., Düzenli, T., (2018). Boundary Analysis for the Derivative of Driving Point Impedance Functions. IEEE Transactions on Circuits and Systems II: Express Briefs, 65, 9, 1149-1153.

[11] Dineen , S., (1989). The Schwarz Lemma. Clarendon Press, USA.

[12] Mercer, P. R., (1997). Sharpened versions of the Schwarz lemma. Journal of Mathematical Analysis and Applications, 205, 2, 508-511.

[13] Mercer, P. R. (2018). Boundary Schwarz inequalities arising from Rogosinski's lemma. Journal of Classical Analysis, 12, 93-97.

[14] Maz'ya, V., Kresin, G., (2007). SharpReal-Part Theorems: A Unified Approach. Springer.

[15] Örnek, B. N., (2015). Carathéodory's inequality on the boundary. The Pure and Applied Mathematics, 2, 2, 169-178.
[16] Örnek, B. N., (2016). The caratheodory inequality on the boundary for holomorphic functions in the unit disc. Journal of Mathematical Physics, Analysis, Geometry, 12, 4, 287-301.

[17] Mercer, P. R., (2018). An improved Schwarz Lemma at the boundary. Open Mathematics, 16, 1, 11401144 .

[18] Osserman, R., (2000). A sharp Schwarz inequality on the boundary. Proceedings of the American Mathematical Society, 128, 12, 3513-3517.

[19] Azeroğlu, T. A., Örnek, B. N., (2013). A refined Schwarz inequality on the boundary. Complex Variables and Elliptic Equations, 58, 4, 571-577.

[20] Boas, H. P., (2010). Julius and Julia: Mastering the art of the Schwarz lemma. The American Mathematical Monthly, 117, 9, 770-785.

[21] Dubinin, V. N., (2004). The Schwarz inequality on the boundary for functions regular in the disk. Journal of Mathematical Sciences, 122, 6, 3623-3629.

[22] Mateljevic, M. (2018) Rigidity of holomorphic mappings \& schwarz and jack lemma, Researchgate. 